\title{
Laparoscopic Management of a Rare Case of Mature Cystic Teratoma With Immature Neural Tissue and Literature Review
}

\author{
Sharon Anqi Foo ${ }^{\text {a, d}}$, Yen Ching Yeo $^{\mathrm{b}}$, Shahul Hameed Mohamed Siraj ${ }^{\mathrm{c}}$
}

\begin{abstract}
Ovarian torsion is one of the most common gynecological emergencies affecting women of reproductive age, in whom prompt diagnosis and timely intervention is vital to preserve ovarian function and mitigate any adverse effect on future fertility. The presence of an ovarian mass is a primary risk factor for ovarian torsion. We present a case of laparoscopic management of ovarian torsion likely secondary to a mature cystic teratoma with immature neural cells. Due to the paucity of such cases reported in the existing literature, we hope that our case will add to the current knowledge and help aid in the counseling and management of future cases.
\end{abstract}

Keywords: Laparoscopic cystectomy; Ovarian torsion; Mature teratoma; Immature cells

\section{Introduction}

Ovarian torsion refers to the complete or partial rotation of the ovary on the infundibulopelvic and utero-ovarian ligaments, often resulting in impedance of its blood supply. It is one of the most common gynecological emergencies [1] and although it may affect females of all ages, the majority of cases affect women of reproductive age, in whom prompt diagnosis and timely intervention is vital to preserve ovarian function and mitigate any adverse effect on future fertility [2]. The presence of an ovarian mass is a primary risk factor for ovarian torsion [2]. Mature cystic teratomas or dermoid cysts are the most common benign germ cell tumors of ovary. They comprise up

Manuscript submitted October 24, 2018, accepted November 12, 2018

aDepartment of General Obstetrics and Gynaecology, KK Women's and Children's Hospital, Singapore

bDepartment of Pathology and Laboratory Medicine, KK Women's and Children's Hospital, Singapore

'Department of Minimally Invasive Surgery, KK Women's and Children's Hospital, Singapore

${ }^{\mathrm{d} C}$ Corresponding Author: Sharon Anqi Foo, 100 Bukit Timah Road, Department of Obstetrics and Gynaecology, KK Women's and Children's Hospital, Singapore 229899, Singapore. Email: sharon.foo@mohh.com.sg

doi: https://doi.org/10.14740/jmc3196 to $30 \%$ of all ovarian tumors [3] and occur most commonly in the second and third decades of life [4] and are bilateral in $10-15 \%$ of patients [5]. The decision to proceed with surgery is based upon a clinical diagnosis made on the combination of symptoms, signs and ultrasound findings. A laparoscopic approach, with its benefit of a shorter hospital stay, less pain and better cosmesis, is typically used [6]. Most torted ovaries are considered potentially viable and removal of any masses via cystectomy with conservation of the ovary is the standard approach for pre-menopausal women.

We present a case of laparoscopic management of ovarian torsion likely secondary to a mature cystic teratoma with immature neural cells. Due to the paucity of such cases reported in the existing literature, we hope that our case will add to the current knowledge and help aid in the counseling and management of future cases.

\section{Case Report}

$\mathrm{Ms} \mathrm{O}$ is a 27-year-old single lady who is virgo intacta. She had a gynecological history of polycystic ovarian syndrome with menstruation every $1-2$ months. She reported normal menstrual volume with no intermenstrual bleed. She had no surgical history.

She presented with right iliac pain, which radiated down her right thigh. It was the first episode of such pain. She did not have any associated fever, nausea or vomiting. She also did not have any urinary or bowel symptoms. She had no abnormal per vagina discharge. The pain was not relieved with opioids.

The patient was afebrile and blood pressure and heart rate were normal.

On examination, the abdomen was soft, with a pelvic mass arising to just below the umbilicus.

\section{Investigations}

A full blood count showed leukocytosis with a total white count of $16 \times 10^{9} / \mathrm{L}$. Liver function test and renal panel were normal.

A transabdominal ultrasound showed a normal uterus with thickened endometrium of $15 \mathrm{~mm}$. A 6-cm avascular left adnexal mass likely compatible with a left ovarian der- 


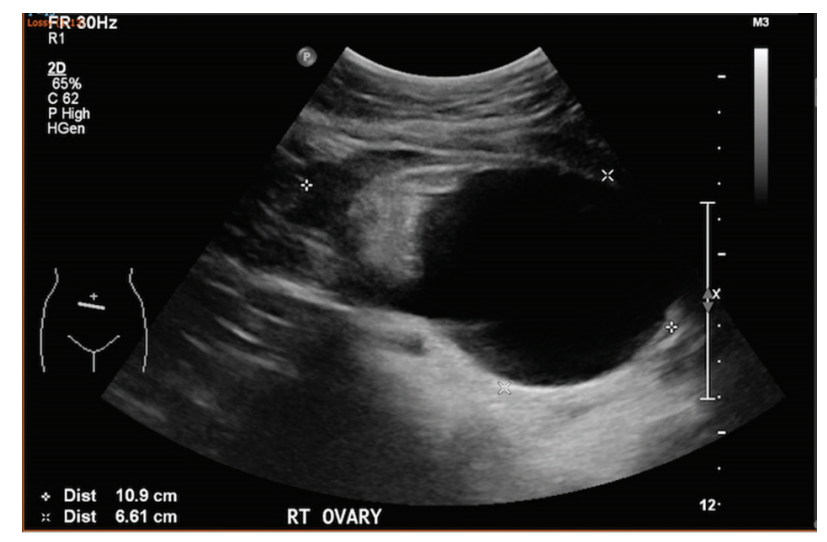

Figure 1. Avascular left adnexal mass of $6.4 \times 3.6 \times 2.3 \mathrm{~cm}$ showed predominantly cystic structure with echogenic components, likely a left ovarian dermoid.

moid was seen (Fig. 1). A large right adnexal cystic structure measuring nearly $10 \mathrm{~cm}$ had surrounding parenchyma which appeared slightly heterogenous and swollen, with no vascularity demonstrated on colour Doppler (Fig. 2). There was also an elongated tubular structure seen just superior to the right ovary which appeared swollen and oedematous, measuring $1.4 \mathrm{~cm}$ in thickness with no intrinsic vascularity demonstrated.

\section{Treatment}

There was high clinical suspicion of ovarian torsion secondary to ovarian cyst, in keeping with her symptoms and scan findings. The patient was offered laparoscopic cystectomy. She was counselled on the risks of intra-operative spillage resulting in chemical peritonitis, risk of loss of normal ovarian tissue implicating future fertility and also that she might require further treatment, including repeat surgery, if the final histology shows malignancy. She was counselled against conservative management due to the risk of tissue necrosis from torsion resulting in loss of viability of the ovary and the deleterious

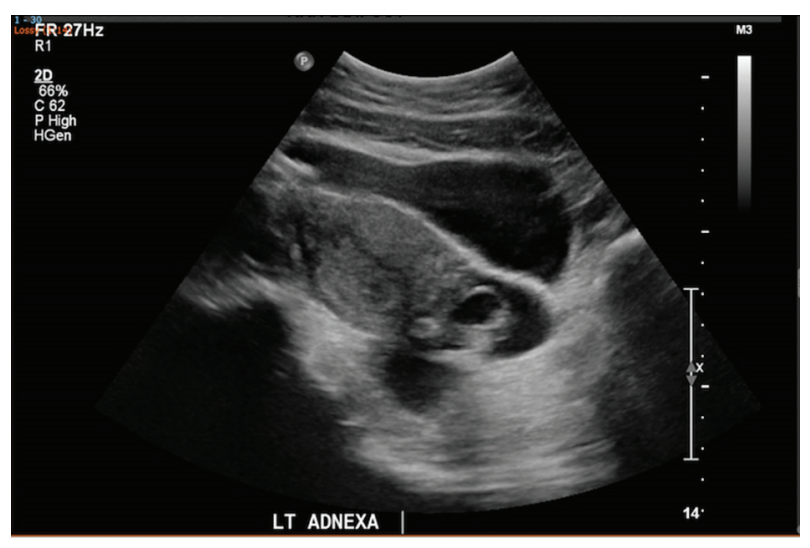

Figure 2. A large right adnexal cystic structure measuring $9.7 \times 9 \times$ $6.2 \mathrm{~cm}$ with avascular echogenic component noted within, likely a right ovarian dermoid.

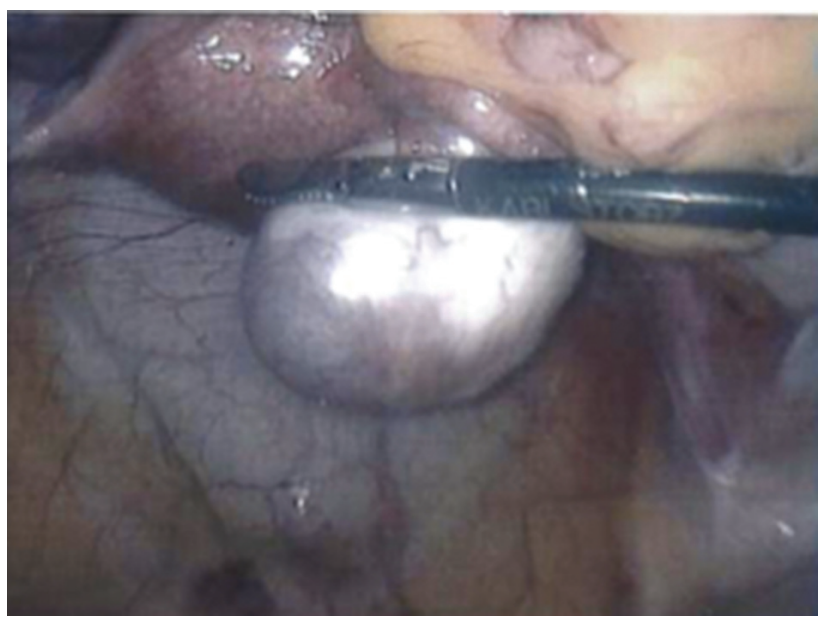

Figure 3. Left ovarian cyst.

effect on future fertility.

She underwent laparoscopic bilateral cystectomy. Intraoperative findings were of a normal uterus with a left bilobed 6-cm cyst (Fig. 3) and a right 9-cm dermoid cyst, which had torted twice on the ovarian pedicle (Fig. 4). Detorsion was done and revascularization of the right ovary was noted (Fig. 5). Bilateral cyst walls were sent for histological evaluation (Fig. 6). The patient recovered well post-operation and was discharged home on the second post-operative day.

\section{Discussion}

The histology of the bilateral cyst walls was initially reported as immature teratoma grade 1 . However, a second opinion reported a final diagnosis of mature cystic teratomas with extensive infarction and microscopic foci of immature neural tissue (Figs. 7, 8). Due to the urgent need for surgical intervention in cases of ovarian torsion, such cases are likely to be undertaken out of hours without the benefit of the histopathology laboratory being able to review a frozen section to aid in on-table

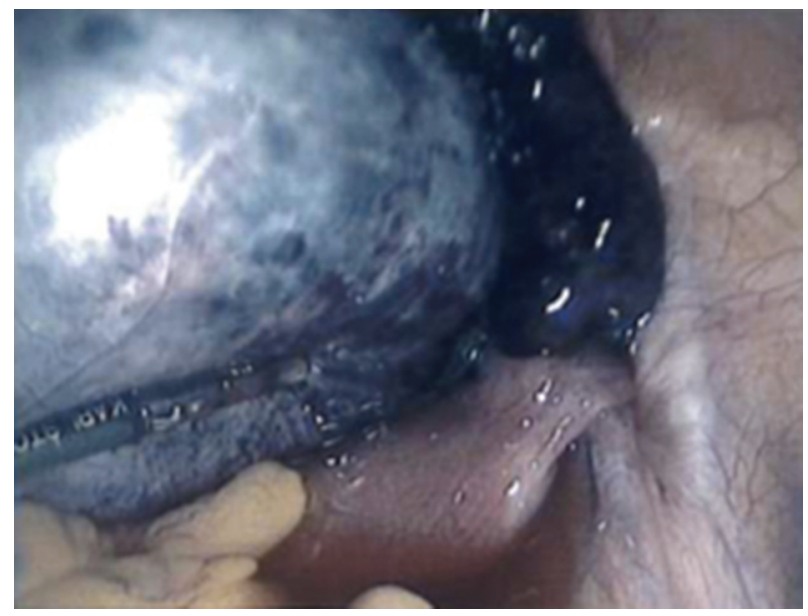

Figure 4. Right ovarian cyst torted around the ovarian pedicle. 


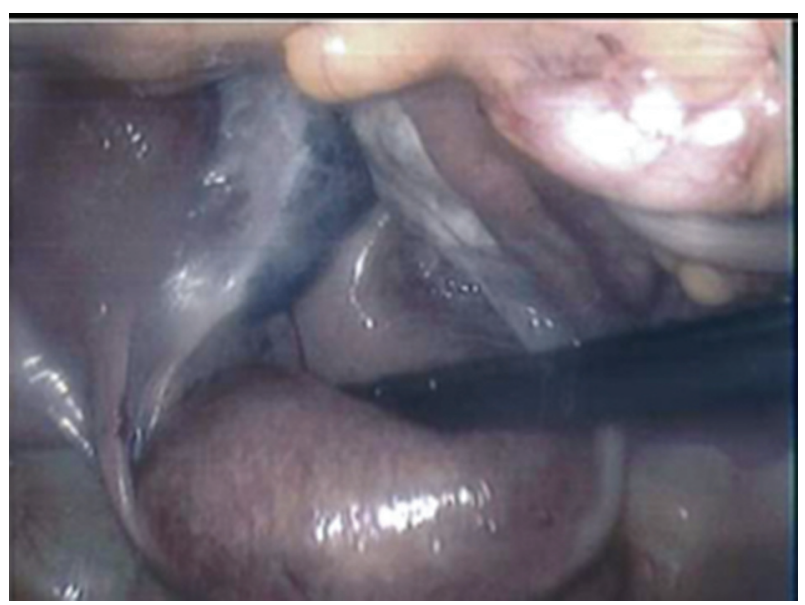

Figure 5. Revascularization of right ovary after detorsion.

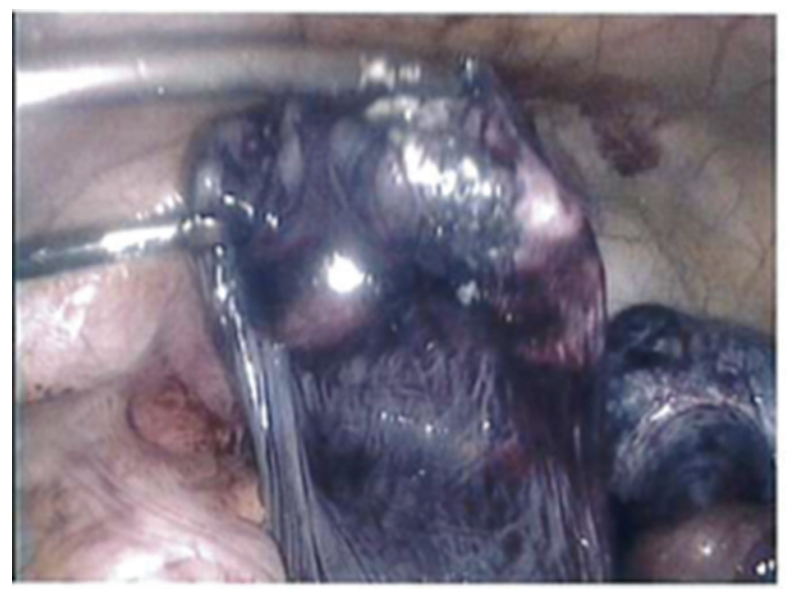

Figure 6. Left ovarian cyst wall.

management of masses suspicious of malignancy. It is also very likely that ovarian masses with the typical appearance of a mature teratoma would not be submitted for a frozen section review. It is only upon final histology that future management would be considered.

A thorough search of the literature revealed the rarity of cases such as ours. A single case report of a patient with an ovarian mature teratoma containing neuroblastomatous tissue showed no recurrence over 7.5 years of follow-up [7]. Dr Yanai-Inbar and Dr Scully reported their experience over a period of 22 years and found 10 patients with immature foci in dermoid cysts [8]. All the cysts had a typical gross appearance. The immature foci ranged from 1 to $21 \mathrm{~mm}$. Seven of the 10 patients had only a cystectomy, two had an oophorectomy and one had a salpingo-opherectomy and hysterectomy. Nine patients were followed from 11 months to 7 years and all were well with no evidence of recurrence. For a young woman in her reproductive years, a histological diagnosis of malignancy can cause much anxiety and result in excessively radical surgery, which would impact future fertility. The good prognosis in existing literature is encouraging, and our patient was counselled accordingly and decision was made for con-

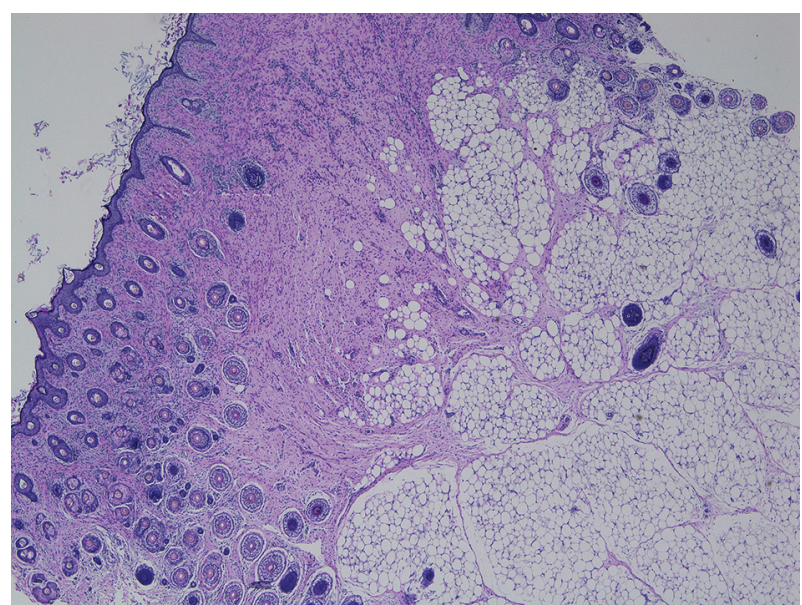

Figure 7. Low power magnification of mature cystic teratoma.

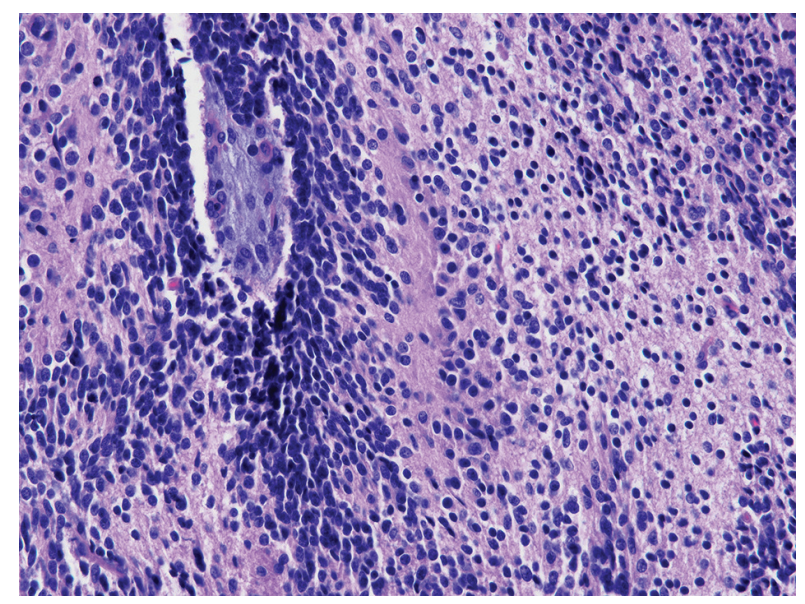

Figure 8. A high power view of immature neural cells.

servative management with ultrasound surveillance for recurrence.

\section{References}

1. McWilliams GD, Hill MJ, Dietrich CS, 3rd. Gynecologic emergencies. Surg Clin North Am. 2008;88(2):265-283, vi.

2. Varras M, Tsikini A, Polyzos D, Samara C, Hadjopoulos G, Akrivis C. Uterine adnexal torsion: pathologic and gray-scale ultrasonographic findings. Clin Exp Obstet Gynecol. 2004;31(1):34-38.

3. Ayhan A, Bukulmez O, Genc C, Karamursel BS, Ayhan A. Mature cystic teratomas of the ovary: case series from one institution over 34 years. Eur J Obstet Gynecol Reprod Biol. 2000;88(2):153-157.

4. Killackey MA, Neuwirth RS. Evaluation and management of the pelvic mass: a review of 540 cases. Obstet Gynecol. 1988;71(3 Pt 1):319-322.

5. Caspi B, Appelman Z, Rabinerson D, Elchalal U, Zalel Y, Katz Z. Pathognomonic echo patterns of benign cystic 
teratomas of the ovary: classification, incidence and accuracy rate of sonographic diagnosis. Ultrasound Obstet Gynecol. 1996;7(4):275-279.

6. Oelsner G, Cohen SB, Soriano D, Admon D, Mashiach $\mathrm{S}$, Carp H. Minimal surgery for the twisted ischaemic adnexa can preserve ovarian function. Hum Reprod. 2003;18(12):2599-2602.

7. Mayberger HW, Carlson AS, Lim S. Immature neural ele- ments (neuroblastomatous change) in benign cystic teratoma: malignant or not? Obstet Gynecol. 1969;33(1):114117.

8. Yanai-Inbar I, Scully RE. Relation of ovarian dermoid cysts and immature teratomas: an analysis of 350 cases of immature teratoma and 10 cases of dermoid cyst with microscopic foci of immature tissue. Int J Gynecol Pathol. 1987;6(3):203-212. 